\title{
Riqueza de mamíferos de grande e médio porte do Parque Nacional da Serra do Divisor (Acre, Brasil)
}

\author{
Armando Muniz Calouro ${ }^{1}$
}

\begin{abstract}
The richness of the large and medium-sized mammals of the Serra do Divisor National Park (Acre, Brazil). The objective of the present study was to characterize the richness (number of species) of large and medium-sized mammals, and the antropic threats in the Parque Nacional da Serra do Divisor (PNSD). In 31 days data were collected through direct observations or evidences (bones, hairs, vocalizations and tracks) along pre-existing trails distributed in different types of vegetation. Wild mammals captured by local people were also considered. They were found 44 species of terrestrial mammals (with the exception of small mammals and bats) and two species of cetaceans, representing $73 \%$ of the total predicted, according to the literature and information of local dwellers were registered in PNSD. They exist in the area two species classified by IUCN (1996) as "Endangered" [Cacajao calvus rubicundus (I. Geoffroy, 1806) and Priodontes maximus (Kerr, 1792)] and five as "Vulnerable" [Ateles chamek (Humboldt,1812), Callimico goeldii (Thomas, 1904), Lagothrix lagotricha poeppigii (Humboldt, 1812), Myrmecophaga tridactyla Linnaeus, 1758 and Inia geoffrensis (Blainville, 1817)]. Given that subsistence and commercial hunting are common in the PNSD, mammals more affected by hunting [Ateles chamek (Humboldt, 1812), Lagothrix lagotricha (Humboldt, 1812), Tayassu pecari (Link, 1795)] were observed only in the more remote areas such as Serra do Divisor, Rio Moa. In comparison with others areas, the results indicate that PNSD has high richness of the mammals, with special attention to the 14 primates species registered.

KEY WORDS. Mammals, geografic distribution, hunting effect, conservation, Acre, Brazil
\end{abstract}

$\mathrm{O}$ estado do Acre insere-se em uma das regiões mais ricas em termos de biodiversidade do globo, estando incluído em um dos "hotspots" citados por MYERS (1988). Apesar de cerca de 30,3\% do Estado do Acre encontrarem-se protegidos em algum tipo de Unidade de Conservação (IMAC 1991), a Estação Ecológica Federal do Rio Acre $\left(775 \mathrm{~km}^{2}\right)$ e o Parque Nacional da Serra do Divisor $\left(8.430 \mathrm{~km}^{2}-5,5 \%\right.$ da área do Estado) são as únicas Unidades de Conservação de Uso Indireto.

O "Workshop 90 - Áreas Prioritárias para a Conservação na Amazônia", realizado em Manaus em 1990, reuniu cerca de 100 pesquisadores de áreas diversas para definirem locais na Amazônia onde o esforço de preservação deveria ser intensificado. Dentre as 94 áreas escolhidas, a região onde se encontra o Parque Nacional da Serra do Divisor (PNSD) foi uma das classificadas dentro do mais alto nível de prioridade (CONSERVATION INTERNATIONAL 1991). Mas apesar de sua relevância biológica, poucos estudos faunísticos foram realizados nas proximidades do PNSD (IHERING 1904; Olalla 1938; VieIRA 1949, 1952; CARVALHO 1957; DAVIS 1973; MoK et. al. 1982; TADDEI et al. 1990; PERES 1997a).

1) Departamento de Ciências da Natureza, Universidade Federal do Acre. Rodovia BR 364 km 04, 69915-900 Rio Branco, Acre, Brasil.

E-mail: calouro@mdnet.com.br 
Esse trabalho objetivou listar a riqueza, ou seja, o número de espécies de mamíferos de grande e médio porte do PNSD com ocorrência confirmada e confrontar com os dados já disponíveis na literatura. Esse trabalho faz parte de uma Avaliação Ecológica Rápida (AER), fruto da parceria existente entre o IBAMA (Instituto Brasileiro do Meio Ambiente e dos Recursos Naturais Renováveis), a SOS-Amazônia e a TNC (The Nature Conservancy), visando coletar o máximo de informações ambientais e sociais que possam subsidiar um Plano de Manejo para o Parque.

\section{MATERIAL E MÉTODOS}

\section{Área de estudo}

O Parque Nacional da Serra do Divisor está localizado no noroeste do Estado do Acre, na divisa com o Peru (Norte $07^{\circ} 07^{\prime} 00^{\prime \prime S}$ e $73^{\circ} 48^{\prime} 20^{\prime \prime} \mathrm{W}$; Leste $09^{\circ} 08^{\prime} 40^{\prime \prime} \mathrm{S}$ e $72^{\circ} 40^{\prime} 00^{\prime \prime} \mathrm{W}$; Sul $09^{\circ} 24^{\prime} 40^{\prime \prime} \mathrm{S}$ e $73^{\circ} 12^{\prime} 40^{\prime \prime} \mathrm{W}$; Oeste $07^{\circ} 32^{\prime} 40^{\prime \prime} \mathrm{S}$ ), possuindo uma área total de 843.012 ha. O PNSD praticamente está dividido em duas partes (Norte e Sul, ligadas por um istmo), com as serras concentradas na porção oeste. Segundo PMACI (1994), as chuvas (2.250 a $2.750 \mathrm{~mm}$ anuais) são freqüentes em praticamente todo o ano, com maior incidência entre novembro e abril. O desnível de altitude verificado entre a serra e as demais regiões determina temperaturas médias anuais entre 23,5 e $25,5^{\circ} \mathrm{C}$. Segundo a classificação de Köppen, o PNSD está inserido em uma área com clima dominante tipo Am (RADAMBRASIL 1977). A região em que está assentado o PNSD é o divisor de águas das bacias hidrográficas do Médio Vale do rio Ucayali (Peru) e do Alto Vale do rio Juruá, e é também onde estão as cabeceiras de vários afluentes pela margem esquerda deste último.

Segundo RADAMBRASIL (1977) existem 10 tipologias florestais no PNSD, sendo que os sítios de coleta de dados foram distribuídos em oito delas:

Tipologia 1 - Floresta Ombrófila Densa Submontana ( $9,18 \%$ da área): Sítio Norte 3: $07^{\circ} 30^{\prime} 22^{\prime \prime} \mathrm{S}$ e $73^{\circ} 42^{\prime} 05^{\prime \prime} \mathrm{W}$ (T1N3).

Tipologia 2 - Floresta Ombrófila Densa dos Interflúvios Colinosos (12,19\%): Sítio Norte 5: 07²7'32"S e 7346'28'W (T2N5)

Tipologia 4 - Floresta Ombrófila Aberta de Palmeiras nas Planícies Aluviais

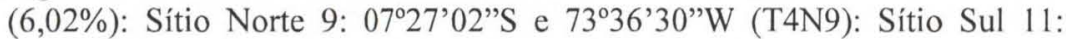
$08^{\circ} 51^{\prime} 37^{\prime \prime} \mathrm{S}$ e $72^{\circ} 52^{\prime} 20^{\prime \prime} \mathrm{W}$ (T4S11).

Tipologia 5 - Floresta Ombrófila Aberta de Palmeiras nos Terraços Altos (4,34\%): Sítio Sul 6: 08³3’30”S e 7253'30”W (T5S6).

Tipologia 6 - Floresta Ombrófila Aberta de Palmeiras nos Depósitos Coluviais $(0,93 \%)$ : Sítio Norte 6: $07^{\circ} 21^{\prime} 23^{\prime \prime} S$ e $73^{\circ} 40^{\prime} 41^{\prime \prime} \mathrm{W}$ (T6N6).

Tipologia 7 - Floresta Ombrófila Aberta de Palmeiras nos Interflúvios Colinosos (34,4\%): Sítio Norte 8: 07³3'24”'S e 73¹6’36”W (T7N8): Sítio Sul 4: $08^{\circ} 24^{\prime} 19^{\prime \prime}$ 'S e $72^{\circ} 51^{\prime} 28^{\prime \prime} \mathrm{W}$ (T7S4).

Tipologia 8 - Floresta O. Aberta nos Interflúvios Colinosos c/ Bambu Dominante (8,57\%): Sítio Sul 1: $08^{\circ} 16^{\prime} 51^{\prime \prime} S$ e $73^{\circ} 15^{\prime} 13^{\prime \prime} \mathrm{W}$ (T8S1).

Tipologia 9 - Floresta O. Aberta nos Interflúvios Colinosos c/ Bambu Dominado (22,97\%): Sítio Sul 4: 0852’31'S e 7246’55'W (T9S4). 


\section{Métodos}

As viagens de campo foram feitas de 05 a 25 de novembro de 1996 e de 06 a 27 de março de 1997, sendo que em cada tipologia procurou-se realizar um esforço mínimo de observação de 15 homem-hora durante o dia e de seis homem-hora durante a noite. Essas observações eram feitas percorrendo-se trilhas já existentes, colhendo-se dados de ocorrência através de observação direta ou por evidências. Essas evidências foram divididas em quatro categorias: pêlos, ossos, vocalizações e rastros (inclui pegadas, trilhas -feitas por pacas, antas e tatus - e tocas). Quando possível foram tirados moldes em gesso das pegadas. De dia, o esforço foi concentrado no horário das 6:00-14:00, sendo que o horário matutino é o mais indicado por ser o período mais ativo dos animais diurnos (BURNHAN et al. 1980). De noite, o esforço foi concentrado no período das 19:00 às 23:00 horas. Foram percorridas, preferencialmente, trilhas já existentes, pouco utilizadas e relativamente retas. A distância máxima do ponto de origem (sempre a margem de um rio) foi de cerca de $9 \mathrm{~km}$, sendo este o caso da trilha que partia do Rio Moa (Tipologia 6). Em média, ficou ao redor de $4 \mathrm{~km}$, próximo da média de $3 \mathrm{~km}$ utilizada em trabalhos similares (Voss \& EMMONS 1996).

Evidências obtidas em outros locais e horários que não os da coleta sistemática por trilhas também foram consideradas, tais como os animais que acabaram de serem caçados, os animais silvestres criados em casa e as evidências obtidas pelas outras equipes de campo. Em todos os sítios de coleta procurou-se coletar ossos, peles e se tirar fotos de animais caçados ou criados nas residências. Todo o material coletado encontra-se depositado na Coleção Zoológica de Mamíferos da Universidade Federal do Acre, Rio Branco, Brasil. Foram entrevistadas 33 famílias da área, aos quais eram mostradas figuras de mamíferos (EMMONS \& FEER 1990; AURICCHIO 1995) para que fossem indicadas as espécies que já haviam sido avistadas na parte do PNSD onde moram e se elas costumam ser caçadas ou não.

$\mathrm{O}$ enfoque metodológico foi dado para mamíferos terrestres de médio e grande porte, excluindo-se portanto os quirópteros, os marsupiais e os pequenos roedores (exceção da família Sciuridae, cujas espécies são avistadas com a metodologia aqui descrita). Para os cetáceos não foram realizadas coletas sistemáticas de informações, mas observações casuais foram anotadas.

Para a identificação das espécies de mamíferos foi utilizada a descrição e a distribuição proposta por EMMONS \& FEER (1990) e ROWE (1996), utilizando-se a nomenclatura existente em WILSON \& REEDER (1993). A nomenclatura das subespécies de primatas foi baseada em PERES (1997a), o qual realizou estimativas de densidade destes animais nas duas margens do Rio Juruá (próximo à foz do Juruá Mirim, mas fora do PNSD).

\section{RESULTADOS}

\section{Número de espécies por tipologias}

Quarenta e três espécies de mamíferos terrestres de grande e médio porte, duas espécies aquáticas (cetáceos) e duas espécies de pequenos mamíferos terrestres foram encontradas no PNSD. Destes pequenos mamíferos, um marsupial foi cole- 
tado e identificado como Marmosa murina (Linnaeus, 1758). Um roedor conhecido como rato-coró (Dactylomys dactylinus (Desmarest, 1817)) foi identificado por sua vocalização pelo ornitólogo Bret M. Whitney. Essas 47 espécies são aquelas cuja ocorrência foi confirmada através de observação direta ou através de outro tipo de evidência (Tab. I).

Tabela I. Espécies de mamíferos de ocorrência confirmada no PNSD.

\begin{tabular}{|c|c|c|}
\hline Ordem & Familia & Nome cientifico \\
\hline Marsupialia & Didelphidae & Marmosa murina (Linnaeus, 1758) \\
\hline \multirow[t]{6}{*}{ Xenarthra } & Bradypodidae & Bradypus variegatus Schinz, 1825 \\
\hline & Dasypodidae & Dasypus novemcinctus Linnaeus, 1758 \\
\hline & & Priodontes maximus (Kerr, 1792) \\
\hline & Megalonychidae & Choloepus spp. ${ }^{1}$ \\
\hline & Myrmecophagidae & Myrmecophaga tridactyla Linnaeus, 1758 \\
\hline & & Tamandua tetradactyla (Linnaeus, 1758) \\
\hline \multirow[t]{14}{*}{ Primates } & Callitrichidae & Callimico goeldii (Thomas, 1904) \\
\hline & & Saguinus f. fuscicollis (Spix, 1823) \\
\hline & & Saguinus imperator subgrisescens (Goeldi, 1907) \\
\hline & & Saguinus mystax mystax (Spix, 1823) \\
\hline & & Cebus albifrons unicolor (Humboldt, 1812) \\
\hline & & Cebus apella (Linnaeus, 1758) \\
\hline & & Saimiri sciureus macrodon (Linnaeus, 1758) \\
\hline & Cebidae & Alouatta seniculus jurua (Linnaeus, 1766) \\
\hline & & Ateles chamek (Humboldt, 1812) \\
\hline & & Lagothrix lagotricha poeppigii (Humboldt, 1812) \\
\hline & & Aotus nigriceps Dollman, 1909 \\
\hline & & Cacajao calvus rubicundus (I. Geoffroy, 1847) \\
\hline & & Callicebus cupreus cupreus (Spix, 1823) \\
\hline & & Pithecia monachus monachus (É. Geoffroy, 1812) \\
\hline \multirow[t]{7}{*}{ Carnivora } & Felidae & Leopardus pardalis (Linnaeus, 1758) \\
\hline & & Panthera onca (Linnaeus, 1758) \\
\hline & & Puma concolor (Linnaeus, 1771) \\
\hline & Mustelidae & Eira barbara (Linnaeus, 1758) \\
\hline & & Lontra longicaudis (Olfers, 1818) \\
\hline & Procyonidae & Bassanicyon alleni Thomas, 1880 \\
\hline & & Potos flavus (Schreber, 1774) \\
\hline \multirow[t]{2}{*}{ Cetacea } & Delphinidae & Sotalia fluviatilis (Gervais \& Deville, 1853) \\
\hline & Platanistidae & Inia geoffrensis (Blainville, 1817) \\
\hline Perissodactyla & Tapiridae & Tapirus terrestris (Linnaeus, 1758) \\
\hline \multirow[t]{4}{*}{ Artiodactyla } & & Cervidae Mazama americana (Erxleben, 1777) \\
\hline & & Mazama gouazoupira (G. Fisher, 1814) \\
\hline & Tayassuidae & Pecari tajacu (Linnaeus, 1758) \\
\hline & & Tayassu pecari (Link, 1795) \\
\hline \multirow[t]{11}{*}{ Rodentia } & Agoutidae & Agouti paca (Linnaeus, 1766) \\
\hline & Dasyproctidae & Dasyprocta fuliginosa Wagler, 1832 \\
\hline & & Myoprocta pratti (Erxleben, 1777) \\
\hline & Dinomyidae & Dinomys branickii Peters, 1873 \\
\hline & Echimyidae & Dactylomys dactylinus (Desmarest, 1817) \\
\hline & Erethizontidae & Coendou prehensilis (Linnaeus, 1758) \\
\hline & Hydrochaeridae & Hydrochaeris hydrochaeris (Linnaeus, 1766) \\
\hline & Sciuridae & Microsciurus flaviventer (Gray, 1867) \\
\hline & & Sciurillus pusilus (Desmarest, 1817) \\
\hline & & Sciurus ignitus (Gray, 1867) \\
\hline & & Sciurus spp. ${ }^{2}$ \\
\hline Lagomorpha & Leporidae & Sylvilagus brasiliensis (Linnaeus, 1758) \\
\hline
\end{tabular}

(1) Possivelmente C. didactylus (Linnaeus, 1758) e C. hoffmanni Peters, 1858 (EMmons 1990);

(2) possivelmente S. spadiceus Olfers, 1818 e S. igniventris Wagner, 1842 (EMmons 1990). 
A tabela II apresenta as espécies distribuídas pelas tipologias florestais e pelos sítios de coleta onde foram encontradas. De forma geral, nota-se que o Sul e o Norte do PNSD apresentaram uma riqueza semelhante de grandes mamíferos (Tab. III). Na Tipologia 2 (no Igarapé Ramon, oeste da Serra da Jaquirana) foi encontrado o maior número de espécies, mas nessa tipologia o tempo de amostra também foi maior (33,3 horas). Ao considerar-se o PNSD como um todo, a Tipologia 7 apresenta o maior número de espécies. Isso é explicado pelo maior tempo de amostra (55,4 horas) e pela heterogeneidade espacial dos sítios de coleta, já que existem espécies que só foram encontradas na Parte Norte e outras na Parte Sul do PNSD.

Tabela II. Distribuição dos grandes mamiferos no PNSD, baseada nas seguintes evidências: (A) avistado, $(R)$ rastro, $(O)$ ossos, $(V)$ vocalização, $(P)$ pêlos.

\begin{tabular}{|c|c|c|c|c|c|c|c|c|c|c|}
\hline \multirow{2}{*}{ Espécies } & \multicolumn{10}{|c|}{ Tipologias } \\
\hline & T1-N3 & T2-N5 & T4-N9 & T6-N6 & T7-N8 & T4-S11 & T5-S6 & T7-S4 & T8-S1 & T9-S10 \\
\hline Myrmecophaga tridactyla & & & & & & & & & $\mathrm{R}$ & \\
\hline Tamandua tetradactyla & & & & $A^{1}$ & & & & A & & \\
\hline Bradypus variegatus & & & & & & & & & $A^{1}$ & \\
\hline Choloepus spp. & $\mathrm{P}$ & $A^{1}$ & & & PO & & & 0 & & \\
\hline Tatus $^{3}$ & R & $\mathrm{R}$ & & $\mathrm{R}$ & $\mathrm{R}$ & & $R$ & $\mathrm{R}$ & $\mathrm{R}$ & $\mathrm{R}$ \\
\hline Dasypus novemcinctus & & & & & $A^{1}$ & & & & $A^{2}$ & \\
\hline Priodontes maximus & $\mathrm{R}$ & $\mathrm{R}$ & & $\mathrm{R}$ & $\mathrm{R}$ & & $\mathrm{R}$ & $\mathrm{R}$ & $\mathrm{R}$ & $\mathrm{R}$ \\
\hline Callimico goeldii & & $A^{1}$ & & & & & & & A & \\
\hline Saguinus f. fuscicollis & A & A & $A^{1}$ & A & A & $A^{1}$ & $A^{1}$ & A & A & $A^{\prime}$ \\
\hline Saguinus imperator subgrisescens & & & & & & $A^{\prime}$ & & & A & \\
\hline Saguinus mystax mystax & A & A & $A^{\prime}$ & A & A & $A^{\prime}$ & A & A & A & A \\
\hline Alouatta seniculus jurua & & A & & & AV & & & & A & \\
\hline Aotus nigriceps & A & A & & A & A & & A & A & & A \\
\hline Ateles chamek & & & & $\mathrm{O}^{2}$ & & & & & & \\
\hline Cacajao calvus rubicundus & & A & & A & & & & & & \\
\hline Callicebus cupreus cupreus & & $A^{1}$ & $\mathrm{v}$ & $\mathrm{v}$ & $\mathrm{v}$ & & & & $\mathrm{v}$ & \\
\hline Cebus albifrons unicolor & & & & & & & $A^{1}$ & $A^{1}$ & A & \\
\hline Cebus apella & A & A & & A & $A^{1}$ & & $A^{1}$ & & 0 & A \\
\hline Lagothrix lagotricha poeppigii & & AV & & AV & & & $A^{1}$ & & & \\
\hline Pithecia monachus monachus & & A & & A & A & & v & & & A \\
\hline Saimiri sciureus macrodon & & $A^{1}$ & & & & & $A^{1}$ & & A & $A$ \\
\hline Gatos-do-mato ${ }^{4}$ & & & & & & & & & & $\mathrm{R}$ \\
\hline Leopardus pardalis & & & & & & & & $\mathrm{P}^{2}$ & & \\
\hline Panthera onca & $\mathrm{R}$ & & & $\mathrm{P}^{2}$ & & & & 0 & & R \\
\hline Puma concolor & & $A^{1}$ & & & & & & & & $\mathrm{R}$ \\
\hline Eira barbara & & & & & A & & & & & \\
\hline Lontra longicaudis & & $A^{2}$ & & & & & & & & \\
\hline Bassaricyon gabbi & & & & A & & & & & & A \\
\hline Potos flavus & & & & A & A & & & $A^{1}$ & & \\
\hline Tapirus terrestris & $\mathrm{R}$ & $R$ & & R & & & & & $\mathrm{R}$ & \\
\hline Mazama americana & $\mathrm{R}$ & $\mathrm{R}$ & & A & $\mathrm{R}$ & & $A R$ & $\mathrm{R}$ & RA & $\mathrm{R}$ \\
\hline Mazama gouazoupira & $P^{2}$ & $\mathrm{R}$ & & & $A^{1}$ & & & $\mathrm{R}$ & $\mathrm{R}$ & $\mathrm{R}$ \\
\hline Pecari tajacu & & AR & & $\mathrm{R}$ & AP & & $\mathrm{R}$ & RO & ARO & $\mathrm{R}$ \\
\hline Tayassu pecari & $\mathrm{R}$ & & & $\mathrm{R}$ & & & & & RO & \\
\hline Agoutipaca & $\mathrm{R}$ & $\mathrm{R}$ & & $\mathrm{R}$ & $\mathrm{R}$ & & $\mathrm{R}$ & $\mathrm{R}$ & $\mathrm{R}$ & $\mathrm{R}$ \\
\hline Dasyprocta fuliginosa & & AR & & $\mathrm{R}$ & A & & AR & $\mathrm{R}$ & A & $\mathrm{R}$ \\
\hline Myoprocta pratti & & & & & $A^{1}$ & & & $\mathrm{R}$ & & AR \\
\hline Dinomys branickii & & $A^{1}$ & & & & & & & & \\
\hline
\end{tabular}


Tabela II. Continuação.

\begin{tabular}{|c|c|c|c|c|c|c|c|c|c|c|}
\hline \multirow{2}{*}{ Espécies } & \multicolumn{10}{|c|}{ Tipologias } \\
\hline & T1-N3 & T2-N5 & T4-N9 & T6-N6 & T7-N8 & T4-S11 & T5-S6 & T7-S4 & T8-S1 & T9-S10 \\
\hline Dactylomys dactylinus & & & & & & $\mathrm{V}^{1}$ & & $v^{1}$ & $V^{1}$ & $\mathrm{~V}^{1}$ \\
\hline Coendou prehensilis & & & $A^{1}$ & & & & & 0 & & $\mathrm{P}$ \\
\hline Hydrochaeris hydrochaeris & & $\mathrm{R}^{2}$ & & & & & & & & \\
\hline Microsciurus flavinter & & & & & & & & A & & \\
\hline Sciurus ignitus & & & & & & & & & A & \\
\hline Sciurus spp. & A & A & & $A$ & A & & A & A & A & A \\
\hline Sciurillus pusilus & & $A^{1}$ & & & & & & & & \\
\hline Sylvilagus brasiliensis & & & & & $A^{1}$ & & & & & \\
\hline Total (44 espécies) & 14 & 25 & 4 & 22 & 21 & 4 & 15 & 22 & 23 & 21 \\
\hline
\end{tabular}

(1) Evidências obtidas por outras equipes de campo; (2) Evidências obtidas pelo autor fora da amostragem por trilhas; (3) Além de Dasypus novemcinctus, os rastros podem pertencer a Cabassous unicinctus e Dasypus kappleri; (4) Além de Leopardus pardalis, as pegadas podem pertencer a Leopardus wiedii e Herpailurus yaguarondi.

Tabela III. Esforço de coleta e distribuição total das espécies pelas tipologias florestais.

\begin{tabular}{ccccc}
\hline Tipologias & Esforço (horas) & Norte & Sul & Total \\
\hline T1 & 22,7 & 14 & - & 14 \\
T2 & 33,4 & 26 & - & 26 \\
T4 & - & 4 & 4 & 6 \\
T5 & 22,1 & - & 15 & 15 \\
T6 & 24,3 & 22 & - & 22 \\
T7 & 55,4 & 21 & 22 & 29 \\
T8 & 22,4 & - & 24 & 24 \\
T9 & 21,5 & - & 21 & 21 \\
\hline Total & 223,2 & 37 & 40 & 44 \\
\hline
\end{tabular}

A alteração dos habitats e a pressão de caça são os principais fatores que podem afetar a ocorrência de diversos mamíferos no PNSD. Assim, para se determinar com maior precisão a riqueza (número de espécies) de mamíferos em cada tipologia seria necessário realizar o trabalho em áreas sem ocupação humana e por um período de tempo maior.

\section{Relatos dos Moradores}

Conforme enquete realizada com 25 famílias da Parte Sul (Tab. IV), existem grupos de Cacajao calvus (I. Geoffroy, 1847), Ateles chamek (Humboldt, 1812) e Lagothrix lagotricha (Humboldt, 1812) na área do Igarapé Reforma e cabeceiras do Igarapé Aparição. Essas áreas apresentariam também uma maior densidade de caça de grande porte, sendo o sítio de caça preferido destes moradores. Alguns moradores desta parte do PNSD costumam caçar Cacajao calvus, Priodontes maximus (Kerr, 1792) e vários carnivoros, ao contrário dos moradores da Parte Norte (sete famílias). Esse hábito pode estar associado a uma menor disponibilidade de caça de maior porte nas proximidades dos rios, obrigando a população a caçar espécies que normalmente não consome. Assim, a redução da caça em certas regiões do Parque pode estar prejudicando também espécies ameaçadas de extinção. Esses relatos indicam a necessidade urgente de se avaliar a pressão de caça e as densidades das espécies aqui listadas. 
Tabela IV. Espécies de mamiferos citados por moradores do PNSD, ressaltando-se as que são caçadas $\left({ }^{*}\right)$.

\begin{tabular}{|c|c|c|c|}
\hline Ordem & Familia & Nome cientifico & Nome local \\
\hline Marsupialia & Didelphidae & Didelphis marsupialis Linnaeus, 1758 & Mucura* \\
\hline \multirow[t]{9}{*}{ Xenarthra } & Myrmecophagidae & Myrmecophaga tridactyla & Tamanduá bandeira \\
\hline & & Ciclopes didactylus (Linnaeus, 1758) & Tamandual \\
\hline & & Tamandua tetradactyla & Mambira \\
\hline & Bradypodidae & Bradypus variegatus & Preguiça \\
\hline & Megalonychidae & Choloepus spp. & Preguiça* \\
\hline & Dasypodidae & Dasypus novemcinctus & Tatu verdadeiro* \\
\hline & & Dasypus kappleri Krauss, 1862 & Tatu açu* \\
\hline & & Cabassous unicinctus (Linnaeus, 1758 ) & Tatu rabo de couro* \\
\hline & & Priodontes maximus & Tatu canastra* \\
\hline \multirow[t]{16}{*}{ Primates } & Callithrichidae & Callimico goeldii & Soim preto* \\
\hline & & Cebuella pygmaea (Spix, 1823) & Leăzinho \\
\hline & & Saguinus $f$. fuscicollis & Soim vermelho \\
\hline & & Saguinus $f$. melanoleucus & Soim branco \\
\hline & & Saguinus imperator & Bigodeiro \\
\hline & & Saguinus mystax & Bigodeiro \\
\hline & & Cebidae Alouatta seniculus & Guariba* \\
\hline & & Aotus nigriceps & Macaco da Noite* \\
\hline & & Ateles chamek & Macaco preto* \\
\hline & & Cacajao calvus rubicundus & Cara de sola/uacari* \\
\hline & & Callicebus cupreus & Zogue* \\
\hline & & Cebus albifrons & Cairara* \\
\hline & & Cebus apella & Macaco prego* \\
\hline & & Lagothrix lagotricha & Barrigudo* \\
\hline & & Pithecia monachus & Parauacu* \\
\hline & & Saimin sciureus & Macaco de cheiro* \\
\hline \multirow[t]{16}{*}{ Carnivora } & Canidae & Atelocynus microtis (Sclater, 1883) & Cachorro do mato* \\
\hline & & Speothos venaticus (Lund, 1842) & Cachorro do mato* \\
\hline & Felidae & Herpailurus yaguarondi (Lacépède, 1809) & Gato preto* \\
\hline & & Leopardus pardalis & Gato maracajá* \\
\hline & & Leopardus wiedii (Schinz, 1821) & Gato açu* \\
\hline & & Panthera onca & Onça pintada* \\
\hline & & Puma concolor & Onça vermelha* \\
\hline & Mustelidae & Eira barbara & Irara \\
\hline & & Galictis vittata (Schreber, 1776) & Furắo* \\
\hline & & Mustela africana Desmarest, 1818 & Doninha \\
\hline & & Lontra longicaudis & Lontra* \\
\hline & & Pteronura brasiliensis (Gmelin, 1788) & Ariranha \\
\hline & Procyonidae & Bassaricyon alleni & Bilibili \\
\hline & & Nasua nasua (Linnaeus, 1766) & Quati* $^{*}$ \\
\hline & & Procyon cancrivorus (G. Cuvier, 1798) & Guaxinim \\
\hline & & Potos flavus & Jupara゙* \\
\hline \multirow[t]{2}{*}{ Cetacea } & Platanistidae & Inia geoffrensis & Boto vermelho \\
\hline & Delphinidae & Sotalia fluviatilis & Tucuxi \\
\hline Perissodactyla & Tapiridae & Tapirus terrestris & Anta* \\
\hline \multirow[t]{4}{*}{ Artiodactyla } & Cervidae & Mazama americana & Veado capoeiro* \\
\hline & & Mazama gouazoupira & Veado roxo* \\
\hline & Tayassuidae & Pecari tajacu & Caititu* \\
\hline & & Tayassu pecan & Queixada* \\
\hline \multirow[t]{11}{*}{ Rodentia } & Agoutidae & Agouti paca & Paca* \\
\hline & Dasyproctidae & Dasyprocta fuliginosa & Cutia* \\
\hline & & Myoprocta pratti & Cutiara* \\
\hline & Dinomyidae & Dinomys branickii & Pacarana* \\
\hline & Echimyidae & Dactylomys dactylinus & Rato coró \\
\hline & Erethizontidae & Coendou prehensilis & Coendu* \\
\hline & Hydrochaeridae & Hydrochaeris hydrochaeris & Capivara* \\
\hline & Sciuridae & Microsciurus flavinter & Quatipuru roxo* \\
\hline & & Sciurillus pusilus & Quatipuru roxo \\
\hline & & Sciurus ignitus & Quatipuru roxo* \\
\hline & & Sciurus spp. & Quatipuru vermelho* \\
\hline Lagomorpha & Leporidae & Sylvilagus brasiliensis & Coelho* \\
\hline
\end{tabular}

Revta bras. Zool. 16 (Supl. 2): 195 - 213, 1999 
Não foi observado nenhum grupo de Saguinus fuscicollis melanoleucus (Spix, 1823) (saguis brancos) dentro do PNSD, somente S. f. fuscicollis (Spix, 1823). Entretanto, todos os moradores da Parte Sul do Parque relataram já terem visto saguis brancos dentro da área. Apesar da distribuição de $S$. $f$. melanoleucus ser restrita à margem direita do Rio Juruá, é muito provável que os moradores tenham observado híbridos destas duas subespécies, com coloração intermediária, como os encontrados por PERES (1996a) na margem esquerda do Rio Juruá. Já Saguinus imperator (Goeldi, 1907) só foi citado pelos moradores da Parte Sul, coincidindo com o observado durante a pesquisa.

A tabela IV deve ser considerada com cautela, sendo somente um forte indicativo da ocorrência das espécies na área, devendo ser confirmada com levantamentos adequados posteriormente.

\section{Descrição das espécies mais ameaçadas}

A tabela V apresenta as espécies mais ameaçadas que ocorrem no PNSD, incluindo tanto aquelas cuja ocorrência foi comprovada, como aquelas cuja ocorrência é provável. As categorias de ameaça são baseadas nos critérios dos Apêndices da Convenção Internacional sobre Comércio de Espécies Ameaçadas (CITES) e da lista de espécies ameaçadas da União Internacional para Conservação da Natureza (IUCN 1996). Para aquelas espécies que não constam da lista de 1996, foi utilizada a classificação da lista de 1994 da IUCN, disponível em FonSECA et al. (1994).

Tabela V. Espécies de grandes mamíferos mais ameaçadas do PNSD (as confirmadas e as de provável ocorrência) conforme luCN (1996) e CITES (Fonte: World Conservation Monitoring Center) e luCn 1994 (FonsECA et al. 1994).

\begin{tabular}{|c|c|c|c|}
\hline Espécie & Nome local & Apêndices da CITES & Classificação da IUCN \\
\hline Choloepus didactylus & Preguiça & III & Insuficientemente conhecida \\
\hline Choloepus hoffmanni & Preguiça & III & Insuficientemente conhecida \\
\hline Myrmecophaga tridactyla & Tamanduá bandeira & $\|$ & Vulnerável \\
\hline Priodontes maximus & Tatu-canastra & I & Em perigo \\
\hline Callimico goeldii & Soim preto & I & Vulnerável \\
\hline Saguinus imperator subgrisescens & Bigodeiro & II & Vulnerável ${ }^{1}$ \\
\hline Ateles chamek & Macaco preto & ॥ & Vulnerável $^{2}$ \\
\hline Cacajao calvus rubicundus & Cara-de-sola/uacari & I & Em Perigo \\
\hline Lagothrix lagotricha poeppigii & Macaco barrigudo & ॥ & Vulnerável \\
\hline Lontra longicaudis enudris & Lontra & I & Vulnerável $^{3}$ \\
\hline Pteronura brasiliensis & Ariranha & i & Vulnerável \\
\hline Mustela africana & Doninha & Não consta & Insuficientemente conhecida \\
\hline Atelocynus microtis & Cachorro-do-mato & Năo consta & Insuficientemente conhecida ${ }^{2}$ \\
\hline Speothos venaticus & Cachorro-do-mato & 1 & Vulnerável \\
\hline Panthera onca & Onça pintada & 1 & Baixo Risco \\
\hline Leopardus wiedii & Gato maracajá & I & Insuficientemente conhecida ${ }^{2}$ \\
\hline Inia geoffrensis & Boto vermelho & II & Vulnerável \\
\hline Sotalia fluviatilis & Tucuxi & 1 & Insuficientemente conhecida \\
\hline Tapirus terrestris & Anta & ॥ & Baixo Risco \\
\hline Dinomys branickii & Pacarana & Não consta & Em perigo $^{2}$ \\
\hline
\end{tabular}

(1)A lucn (1996) considera vulnerável apenas a subespécie Saguinus i. imperator, (2) presente apenas na lista da IUCN (1994) (FONSECA et al. 1994); (3) presente apenas na lista da IUCN (1994), onde considerava vulnerável somente Lontra I. longicaudis.

Das espécies listadas como insuficientemente conhecidas pela IUCN, vale a pena comentar Mustela africana Desmarest, 1818. Esse é um dos mamíferos menos conhecidos dos neotrópicos: possui uma distribuição pouco definida pela 
Amazônia, é semi-aquático e aparentemente raro, o que pode indicar uma exigência de habitat (FONSECA et al. 1994). Existe um registro em ORCES (1944) na margem esquerda do Rio Juruá, no Acre, mas não fica claro se dentro ou fora do PNSD (FONSECA et al. 1994). A seguir uma breve descrição das espécies mais ameaçadas.

\section{Ateles chamek}

Baseado na distribuição da espécie (Rowe 1996), provavelmente o macacopreto que ocorre no PNSD é Ateles chamek, mas não foi observado nenhum animal diretamente para confirmar a identificação. Houve somente uma evidência de sua ocorrência no PNSD: um crânio encontrado próximo ao Igarapé Anil (cerca de 8km do Rio Moa - T6N6), de um animal caçado por um morador da área a cerca de três meses.

Basicamente frugívoro, com baixas taxas reprodutivas, necessidade de grandes áreas de vida e grupos divididos em pequenos subgrupos (ROOSMALEN \& KLEIN 1988), Ateles é facilmente extinto localmente mesmo sob uma pressão de caça moderada, sendo muito visado pelo seu tamanho (PEREs 1990). Esse animal só deve ocorrer no PNSD a grandes distâncias das margens dos rios mais habitados e, preferencialmente, em florestas densas, altas e pouco perturbadas.

\section{Cacajao calvus rubicundus (I. Geoffroy, 1806)}

Foram observados dois grandes grupos de uacaris vermelhos no PNSD. ambos com mais de 40 indivíduos: um próximo ao Igarapé Anil (T6N6) e o outro ao Igarapé Ramon (T2N4), na margem direita. Vale ressaltar que ambos os grupos foram observados próximos a grandes igarapés, em vegetação densa de grande porte. RYLANDS (1991) coloca que Cacajao calvus ucayali poderia ocorrer no PNSD, mas os indivíduos observados possuem características morfológicas de Cacajao calvus rubicundus.

Existem poucas informações disponíveis sobre a ecologia dessa subespécie de Cacajao, sendo o gênero muito ameaçado pela sua distribuição restrita e pela pressão de caça (FONSECA et al. 1994). Apesar de sofrer pressão de caça por parte de alguns moradores (especialmente no Sul do PNSD), sua distribuição dentro do Parque pode estar mais relacionada com uma exigência de habitat (florestas densas, sobre solos férteis e com grande disponibilidade de frutos) do que com uma perturbação qualquer. Pelos informes dos moradores, na Parte Sul do PNSD a espécie ocorreria principalmente em áreas pouco habitadas (cabeceira do Igarapé Aparição, próximo ao sopé da Serra do Rio Branco). Considerando-se que só existem registros de ocorrência desta subespécie em apenas outras duas unidades de conservação (FONSECA et al. 1994), deve ser considerado prioritário um estudo para avaliar o status populacional desta espécie e a real distribuição da mesma dentro do PNSD, devido ao grau de ameaça existente para Cacajao calvus rubicundus em outras regiões não protegidas.

\section{Callimico goeldii (Thomas, 1904)}

Naturalmente rara e com distribuição restrita no Brasil ao Estado do Acre e ao trecho do Rio Juruá no Estado do Amazonas, Callimico goeldii é uma espécie com uma exigência muito forte de habitat: prefere tipologias florestais com subbosque denso e com ocorrência de bambu (PoOK \& POOK 1981). No PNSD foi 
observado um grupo em uma área de capoeira com sub-bosque denso sem bambu no Igarapé Ouro Preto (T7S4) e outro encontrado em uma mancha de bambu no Igarapé Ramon (T2N5). Essa espécie, devido ao seu pequeno porte, não costuma ser alvo dos caçadores locais.

\section{Lagothrix lagotricha poeppigii}

Apesar de ter se adotado aqui a subespécie Lagothrix lagothricha poeppigii, como proposto por RYLANDS (1991), não é seguro afirmar que essa é a identificação correta para os animais observados no PNSD. Variações na coloração (mais próximas de L.l. cana) foram observadas em campo e confirmadas pelos moradores, variações nem sempre coincidentes com a esperada para Lagothrix lagothricha poeppigii.

O macaco-barrigudo apresenta várias características em comum com Ateles: tem uma dieta basicamente frugívora, sendo um importante dispersor de grandes sementes; possui baixa taxa reprodutiva; prefere florestas primárias altas e densas e é muito sensível à pressão de caça (RAMIREZ 1988). No PNSD, entretanto, Lagothrix parece estar em uma situação populacional mais favorável que Ateles. Foram observados quatro grupos na T6N6 (dois deles com mais de 20 indivíduos) e dois na T2N5.

\section{Saguinus imperator subgrisescens}

Espécie de primata com distribuição no Brasil muito semelhante a de Callimico goeldii: praticamente todo o Estado do Acre e a região fronteiriça do Estado do Amazonas (Rowe 1996). S. i. subgrisescens aparentemente não é comum no PNSD, sendo que somente quatro grupos foram encontrados, todos na Parte Sul do Parque. Já Saguinus fuscicollis e, principalmente, Saguinus mystax foram encontrados com muita freqüência (este último em praticamente todas as tipologias do Parque), inclusive nos mesmos locais em que foram observados os grupos de S.i.subgrisescens. Vale ressaltar que em nenhum momento foi observado um grupo isolado de $S$. fuscicollis, sendo sempre encontrado formando grupos mistos com $S$. mystax.

\section{Dinomys branickii Peters, 1873}

Com hábitos pouco conhecidos, naturalmente raro na natureza, esse roedor possui uma distribuição restrita ao oeste da Amazônia, ocorrendo no Brasil no Acre, Rondônia, sudoeste do Amazonas e, provavelmente, no Mato Grosso (EMMONs \& FEER 1990). É caçado pelos moradores do PNSD (Tab. IV), sendo que houve um único registro de um indivíduo que havia acabado de ser caçado.

Considerada em perigo pela IUCN em 1994, a espécie sofre riscos com a destruição do habitat e a pressão de caça. Sua raridade natural e o desconhecimento de sua ecologia favorecem sua inclusão nessa categoria, pois não existem estudos que mostrem sua susceptibilidade à perturbações ambientais.

\section{Tapirus terrestris (Linnaeus, 1758)}

Apesar de sua ampla distribuição natural, Tapirus terrestris já desapareceu de diversas regiões. Devido ao seu porte, é muito visada pelos caçadores. Seus hábitos semi-aquáticos favorecem a sua captura: costuma ser perseguida com o auxílio de cães, indo refugiar-se nos igarapés, onde é facilmente abatida. Graças 
também à suas baixas taxas reprodutivas, Tapirus terrestris é considerada a espécie mais sensível à pressão de caça entre os ungulados neotropicais (BODMER et al. 1988a). Dentro do PNSD, a maior parte dos registros ocorreram na área menos habitada da Parte Norte, onde ainda é possível observar rastros destes animais próximo das margens do Rio Moa e Igarapé Ramon. No restante do Parque, a espécie só deve ocorrer a grandes distâncias das margens dos rios e igarapés habitados.

\section{Lontra longicaudis (Olfers, 1818)}

Esta espécie foi observada em duas ocasiões no PNSD, mas somente no Igarapé Ramon, região oeste da Serra da Jaquirana. L. longicaudis é uma espécie carnívora semi-aquática, que evita áreas com ocupação humana, sendo muito sensível à variações na qualidade da água (FonsECA et al. 1994). Como a população humana no PNSD está concentrada nas margens de rios e igarapés, provocando perturbações nestes ambientes, a ocorrência de L. longicaudis nestes locais se torna mais improvável quanto maior for o grau de perturbação. Ainda segundo FONSECA et al. (1994), essa espécie está associada a ambientes com substratos duros, que formem costões rochosos onde o animal possa se abrigar (ambientes existentes no Igarapé Ramon). Assim, pelas características ambientais do PNSD e pelas exigências de habitat da espécie, é possível que L. longicaudis tenha sua distribuição restrita dentro do PNSD às áreas pouco habitadas e adjacentes às Serras da Jaquirana, Moa e Rio Branco.

\section{Pteronura brasiliensis (Gmelin, 1788)}

Não houve registros dessa espécie no PNSD, mas sua ocorrência é provável pelo Parque estar inserido em sua área de distribuição (EMMONS \& FEER 1990) e pelos relatos dos moradores (Tab. IV). Apesar de sua ampla distribuição, já está extinta em várias partes do país. Populações maiores da espécie encontram-se principalmente na bacia amazônica, mas mesmo aqui suas densidades estão em declínio pela caça, alteração da vegetação ribeirinha e da qualidade da água (FonSECA et al. 1994). Dentro do PNSD, conforme relato de moradores, Pteronura brasiliensis ocorre somente em áreas pouco habitadas.

\section{Speothos venaticus (Lund, 1842)}

Não houve registros dessa espécie no PNSD, mas sua ocorrência é provável pelo Parque estar inserido em sua área de distribuição (EMMONS \& FEER 1990) e por relatos dos moradores (Tab. IV). A confirmação de sua ocorrência no PNSD é importante, pois a espécie é naturalmente muito rara, pouco estudada na natureza e, aparentemente, é muito sensível a alterações do habitat e redução no número de suas presas (FONSECA et al. 1994).

\section{Panthera onca (Linnaeus, 1758)}

Predador de topo de cadeia, as principais perturbações que ameaçam a sobrevivência da Panthera onca na natureza são a destruição do habitat e a pressão de caça. Como necessitam de grandes áreas de vida para sobreviver (QUIGLEY \& CRAWSHAW 1992), a existência de grandes populações restringe-se hoje à floresta amazônica. Algumas das evidências no PNSD são de animais que foram caçados pelos moradores, sendo que esta pressão de caça deve ser considerada atualmente a principal ameaça para a espécie dentro do PNSD. 


\section{Priodontes maximus}

O tatu-canastra é o maior tatu existente, estando em perigo de extinção por causa da caça e da destruição do habitat, apesar de sua ampla distribuição. Apesar de naturalmente raro, no PNSD foram observadas várias tocas recentes destes animais na maioria das tipologias amostradas (Tab. I), o que indica que a espécie apresenta uma população razoável na área. Apesar de outras espécies de tatus serem caçadas na região, o tatu-canastra aparentemente não é muito consumido pela população local, principalmente na Parte Norte. Em conversas com moradores, foi observada a existência de tabu alimentar de origem cultural: matar esse animal daria azar para o caçador. Esse tipo de tabu é conhecido como panema e faz parte da cultura regional, existindo para várias outras espécies (GALVÃo 1951).

\section{Myrmecophaga tridactyla Linnaeus, 1758}

Apesar de ocorrer em todo o Brasil, as maiores densidades populacionais da espécie estão nos Cerrados do Planalto Central. Com a substituição deste tipo de vegetação por monoculturas, com a intensificação do uso do fogo e com a caça predatória, Myrmecophaga tridactyla encontra-se hoje ameaçado no seu principal habitat (FonsECA et al. 1994). Estudos sobre o status da espécie na região amazônica ainda não foram realizados. Dentro do PNSD, foram registradas pegadas de dois animais diferentes em uma única tipologia da Parte Sul.

\section{Sotalia fluviatilis (Gervais \& Deville, 1853) Inia geoffrensis (Blainville, 1817)}

Dentro do PNSD esses cetáceos foram observados nos seguintes locais: Sotalia fluviatilis - um indivíduo na foz do Rio Azul, um indivíduo no Rio Moa, dois indivíduos na foz do Rio Juruá-Mirim; Inia geoffrensis - um indivíduo na foz do Rio Juruá Mirim. Como não foi utilizada uma metodologia específica para observar esses animais, esses dados servem apenas como evidência da ocorrência das duas espécies no PNSD. Considerando-se o status dado para esses cetáceos pelo IUCN (1996) (Tab. V), estudos posteriores devem ser realizados para esclarecer a situação populacional dos mesmos no PNSD, principalmente Inia geoffrensis.

\section{DISCUSSÃO}

\section{Riqueza de mamíferos do PNSD}

A fauna de mamíferos da Amazônia Ocidental provavelmente é uma das mais ricas do mundo, devido a diversos fatores, tais como: (a) presença de rios meândricos que causam processos erosivos nas margens e a formação de mosaicos de vegetação; (b) maior pluviosidade da Amazônia e alta incidência de radiação solar, gerando alta produtividade primária; (c) assincronismo na produção de frutos, ou seja, a disponibilidade de alimento não é sazonal; (d) área de rios de água branca, ricos em nutrientes provenientes dos Andes que são parcialmente depositados nas várzeas (Voss \& EMMONS 1996).

Através da literatura e da enquete feita com os moradores, pode-se estimar a existência de cerca de 63 espécies e subespécies de grandes mamíferos no PNSD (Tab. IV). Logicamente, somente estudos mais específicos e de longo prazo poderão 
tornar essa estimativa mais realista. Mas tomando-se esse número como a riqueza esperada, as 46 espécies encontradas [desconsiderando Marmosa murina (Linnaeus, 1758)] representariam 73\% do total. Segundo Voss \& EMMONS (1996), a diversidade real está oscilando cerca de $\pm 10 \%$ da diversidade esperada de uma área (os autores usam o conceito de diversidade de espécies como sinônimo de riqueza). Sendo assim, a diversidade encontrada de mamíferos no PNSD está entre $67 \%$ e $81 \%$ da diversidade real.

A comparação dos resultados encontrados no PNSD com outras áreas é problemática considerando-se as diferenças metodológicas para obtenção dos dados, o tempo de coleta, as variações naturais de uma área para outra, a ocupação humana e a experiência profissional dos pesquisadores envolvidos em cada área (EMMONS 1984; Voss \& EMMONS 1996). Tendo essas premissas em mente, os dados da tabela VI demonstram que a diversidade de mamíferos do PNSD é realmente elevada, considerando-se o pouco esforço de coleta feito no Parque e o fato de que os pequenos mamíferos ainda não foram inventariados.

Tabela VI. Número de espécies de mamiferos terrestres registradas em 11 localidades neotropicais (adaptado de Voss \& EMMONS 1996).

\begin{tabular}{lccccccccc}
\hline \multicolumn{1}{c}{ Localidades } & Marsup. & Xenarth. & Quirop. & Primat. & Carniv. & Ungul. ${ }^{1}$ & Roden. & Lagom. & Total \\
\hline PNSD (Brasil) & 1 & 6 & $55^{2}$ & 14 & 7 & 5 & 11 & 1 & 100 \\
La Selva (Costa Rica) & 5 & 7 & 65 & 4 & 14 & 5 & 16 & 1 & 117 \\
Barro Colorado (Panamá) & 6 & 6 & 64 & 4 & 13 & 5 & 14 & 1 & 113 \\
Kartabo (Guiana) & 7 & 9 & - & 6 & 13 & 5 & 20 & 0 & $(60)$ \\
Arataye (Guiana Francesa) & 9 & 8 & 61 & 7 & 11 & 5 & 21 & 0 & 122 \\
Cunucunuma (Guiana) & 8 & 7 & 50 & 7 & 7 & 3 & 11 & 0 & 93 \\
Reservas MCSE (Brasil) & 9 & 8 & - & 6 & 8 & 5 & 17 & 0 & $(53)$ \\
Xingu (Brasil) & 8 & 4 & 47 & 7 & 2 & 3 & 23 & 1 & 95 \\
Balta (Peru) & 11 & 9 & 56 & 10 & 15 & 4 & 24 & 1 & 130 \\
Cocha Cashu (Peru) & 12 & 7 & 60 & 13 & 14 & 5 & 27 & 1 & 139 \\
Cuzco Amazónico (Peru) & 9 & 5 & 44 & 7 & 11 & 4 & 22 & 1 & 103 \\
\hline
\end{tabular}

(1) Inclui Artiodactyla e Perissodactyla; (2) Dados do levantamento de quirópteros (Marcelo R. Nogueira, André Pol, André M. da Silva e Carolina P.H. Rocha).

\section{Densidade humana e a pressão de caça}

A riqueza de mamíferos em uma área é determinada por uma série de fatores. No caso do PNSD, a caça de subsistência e a caça comercial podem estar afetando a variedade de espécies de grandes mamíferos nas diferentes tipologias. Algumas espécies são muito sensíveis à pressão de caça, desaparecendo rapidamente (ex: Ateles, Lagothrix) ou mais lentamente (ex: Tayassu pecari (Link, 1795), Tapirus terrestris), ou apenas tendo suas densidades reduzidas na área(BODMER et al. 1988a, 1995; PEREs 1990, 1996b, 1997b). Essa redução nas densidades das espécies caçadas pode provocar verdadeiros "vazios" dentro da floresta, com conseqüências ecológicas ainda pouco conhecidas (REDFORD 1992). A densidade humana em uma área indica indiretamente a pressão da caça de subsistência que a fauna local está sofrendo. Já a caça comercial exerce um efeito muito mais drástico, reduzindo rapidamente as populações das espécies caçadas.

Conforme informações preliminares do Levantamento Sócio-Econômico realizado no PNSD em 1997, a densidade humana no PNSD é de cerca de 0,68 
habitantes $/ \mathrm{km}^{2}$ (3937 moradores na Parte Sul e 1848 moradores na Parte Norte, incluídos os ribeirinhos que moram nas margens que ficam fora do PNSD). Em outras áreas da Amazônia onde foram constatadas diminuições das populações de algumas espécies animais devido à pressão de caça, a densidade humana variou de 0,01 a 0,62 habitantes $/ \mathrm{km}^{2}$ (AYRES \& AYRES 1979; BODMER et al. 1988b; VICKERS 1984, 1991; PERES 1990). Assim, a densidade humana existente no PNSD sugere que uma pressão de caça expressiva deve estar ocorrendo na área.

Entretanto, a distribuição destas pessoas no PNSD é que pode fornecer um melhor quadro dos pontos onde a pressão de caça deve ser mais acentuada. Em primeiro lugar, os moradores do PNSD são todos ribeirinhos, não ocupando as regiões centrais do Parque. PERES \& TERBORGH (1995) estimam que o raio de ação dos caçadores não ultrapassa $10 \mathrm{~km}$ do ponto de origem, ou seja, $10 \mathrm{~km}$ das margens dos rios no PNSD. Pela distribuição das famílias na área é possível determinar alguns locais onde o acesso é, teoricamente, mais difícil. Além das próprias serras, pode-se listar na Parte Norte: (a) o lado oeste da Serra da Jaquirana e da Serra do Moa (regiões desocupadas nas Tipologias 1 e 2); (b) o lado leste da Serra do Moa, onde somente a cerca de $22 \mathrm{~km}$ da Serra encontram-se moradores vivendo no Paraná Novo Recreio (Tipologia 7), mas essa região pode ser atingida pelo Igarapé José Grande (afluente do Rio Azul) e o próprio Paraná Novo Recreio; (c) as cabeceiras dos rios Azul e Juruá-Mirim, no istmo que liga o Norte ao Sul do PNSD (Tipologias 1 e 2).

A Parte Sul do PNSD possui muitos igarapés, facilitando o acesso ao interior do PNSD. Fora os moradores que vivem às margens do Rio Juruá, a maior parte dos ribeirinhos vive nas margens do Rio das Minas e do Igarapé Ouro Preto (dados preliminares do Levantamento Sócio-Econômico). As áreas desocupadas e supostamente menos caçadas são: (a) a região ao leste da Serra do Rio Branco (Tipologias 1 e 8), acessível pelo Rio Branco, Igarapé Ouro Preto e Rio Belo; (b) a região ao sul do Igarapé Paratari, com poucos moradores, englobando o Igarapé São Luís e a microbacia do Igarapé Aparição (Tipologias 8 e 9).

Esses locais menos afetados pela ação antrópica podem estar servindo como fonte de dispersão de animais para o restante do PNSD e devem ser considerados como áreas intocáveis no Plano de Manejo. Porém, Peres \& Terborgh (1995) enfatizam que igarapés pequenos, que não aparecem nas imagens de satélite, podem servir como rota de entrada para esses locais, pelo menos no período chuvoso. Outro fator a ser investigado futuramente é se os moradores do PNSD costumam realizar expedições de caça e, principalmente, onde e com que freqüência. Em conversas informais durante a viagem, alguns caçadores confirmaram realizar tais expedições, com vários dias de duração.

Conforme os dados fornecidos pelo Levantamento Sócio-Econômico, cerca de $67 \%$ das famílias do PNSD concentram-se ao longo dos rios Juruá e Juruá-Mirim, ou seja, na Parte Sul do Parque. Apesar das diferenças ambientais, essa concentração humana explica em parte a diferença na composição de espécies observadas da Parte Norte para a Parte Sul. A menor ação antrópica permitiu que espécies indicadoras (Lagothrix, Panthera, Tapirus, Tayassu pecari, Lontra) fossem observadas no curto espaço de tempo deste trabalho, o que é um forte indicativo que a Parte Norte apresenta-se menos perturbada que a Parte Sul. Das espécies só encontradas na Parte 
Sul merecem destaque Saguinus imperator subgrisecens e Dactylomys dactylinus (Desmarest, 1817), sendo que este último é um roedor típico de vegetações com bambu.

\section{CONSIDERAÇÕES FINAIS}

Pelos resultados obtidos, pode-se colocar o PNSD como uma das Unidades de Conservação de maior riqueza de grandes mamíferos dos Neotrópicos. Levantamentos detalhados da quiropterofauna e dos pequenos mamíferos terrestres devem ser feitos para comprovar a veracidade dessa afirmação para todos os mamíferos. Além desses levantamentos, os dados coletados nesse trabalho permitem listar também outras três linhas prioritárias de pesquisa na área:

\section{Avaliação da Pressão de Caça e da sua Sustentabilidade}

Quando considera-se a Parte Norte do PNSD, a caça aparentemente não afetou de forma intensa a riqueza de grandes mamíferos, pois foi possível encontrar espécies que desaparecem rapidamente quando sob pressão de caça, tais como Ateles, Lagothrix, Tapirus, Tayassu pecari (BODMER et al. 1988a, 1995; PERES 1990, 1996b). Esse quadro é diferente na Parte Sul do PNSD, onde essas espécies não foram avistadas e a densidade humana é maior. Infelizmente, existe um tipo de pressão de caça que está atingindo o PNSD como um todo. Como exemplo é possível citar a caça comercial sistemática feita por caçadores de Cruzeiro do Sul (AC) no Rio Moa, para venda da carne destes animais nos restaurantes daquela cidade. Foi constatado também que comerciantes peruanos compram periodicamente peles de Pecari tajacu de moradores do Rio Juruá Mirim. Assim, a caça comercial está se tornando uma nova fonte de renda para a população local e da região, tendo em vista a falta de alternativas econômicas frente à falência do extrativismo da borracha.

Uma avaliação da sustentabilidade da caça que ocorre no PNSD se faz necessária, pois os efeitos que a redução das densidades das espécies caçadas podem estar causando ao ecossistema não são bem conhecidos, mas inferidos pelo fato de grande parte dos animais afetados terem sua dieta baseada no consumo de frutos e sementes, funcionando como dispersores e predadores de sementes (REDFORD 1992).

\section{Preservação de Primatas}

Foi constatada a ocorrência de 14 espécies de primatas no PNSD, podendo chegar a 15 se incluirmos Cebuella pygmaea (Spix, 1823). Segundo RYLANDS (1991), somente outras três Unidades de Conservação na Amazônia Brasileira teriam mais espécies de primatas: a Reserva Biológica do Abufari, Amazonas (19 espécies), o Parque Nacional da Amazônia, Pará (16 espécies) e a Reserva Ecológica Jutaí-Solimões, Amazonas (16 espécies). RYLANDS (1991) lista 13 espécies para o PNSD, não incluindo Cacajao calvus, Cebuella pygmaea e Saguinus imperator, mas citando duas prováveis espécies de Callicebus na área (C. caligatus (Wagner, 1842) e C. cupreus cupreus). Infelizmente, Callicebus não foi observado durante este trabalho, mas registrado por sua vocalização característica. Assim, foi adotado somente Callicebus cupreus cupreus, conforme observado por PERES (1997a) na foz do Rio Juruá-Mirim. 
A área do entorno do PNSD apresenta espécies e subespécies de primatas que, aparentemente, não ocorrem no seu interior (Pithecia irrorata Gray, 1842, Saguinus fuscicollis melanoleucus, Saimiri boliviensis (I. Geoffroy \& Blainville, 1834) e Cebuella pygmaea, todos observados por PERES (1997a) na margem direita do Rio Juruá). Com a existência do Parque, existem limitações legais para certas atividades neste entorno. Por isso é interessante que o Plano de Manejo da área sugira estratégias para manter parte da cobertura florestal do entorno, baseando-se nas normas legais já existentes referentes à manutenção de mata ciliar e do percentual que não pode ser desmatado em cada propriedade particular.

Por estar classificado como "Em Perigo de Extinção" pela IUCN e por estar sofrendo pressão de caça dentro do PNSD, Cacajao calvus rubicundus, sem dúvida, merece um estudo urgente para avaliar sua situação na área.

\section{Preservação de Felinos}

Os moradores do PNSD costumam matar felídeos, principalmente os de maior porte, como a onça-pintada (Panthera onca). A razão alegada é que esses carnívoros estariam matando os animais de criação, sendo necessário o seu abate. QUIGLEY \& CRAWSHAW (1992) registraram a ocorrência deste tipo de comportamento para Panthera onca no Pantanal, atacando principalmente bezerros. Como a dieta destes predadores é baseada em presas que também são comumente caçadas pelos moradores (RABINOWITZ \& NOTTINGHAM 1986; EMMONS 1987), talvez a pressão de caça (principalmente a comercial) esteja diminuindo a quantidade de presas para os felinos, obrigando-os a utilizarem outras fontes (QUIGLEY \& CRAWSHAW 1992; WEBER \& RABINOWITZ 1996). Por outro lado, o fato dos animais domésticos serem criados soltos e adentrarem a floresta freqüentemente (foram observados rastros de porcos domésticos a mais de $300 \mathrm{~m}$ da moradia) pode favorecer a predação por parte dos felinos, independente da disponibilidade de presas silvestres ter diminuído ou não. Um outro motivo é o medo que os caboclos amazônicos possuem dos grandes felídeos. Assim, na ótica do ribeirinho, é melhor matar esses animais do que conviver com eles.

Os grandes carnívoros desempenham um importante papel no controle das populações de suas presas e a ocorrência destes animais no PNSD é um indicativo de que o ecossistema está pouco perturbado. Entretanto, são necessárias pesquisas de ecologia das espécies, que avaliem o tamanho e a distribuição das populações dos felinos na área, a viabilidade destas se manterem estáveis no longo prazo e a disponibilidade de presas para manter essas populações. Além disso, deve ser averiguada a gravidade do conflito existente entre os felídeos e os moradores da área e do entorno (competição por presas, predação de animais domésticos e aspectos culturais), a fim de serem formuladas propostas para amenizar estes problemas.

AGRADECIMENTOS. Agradeço aos pesquisadores Bret Whitney, Elder Morato, Letícia Santos, Lindomar Soares, Moisés Barbosa, Rosemary Vieira, Rosimeire Correia e Verônica Passos pelo interesse em repassar informações que enriqueceram esse trabalho; ao técnico Willian Aiache pelo auxílio no tratamento do material coletado; à Júlio Bicca-Marques pela

Revta bras. Zool. 16 (Supl. 2): 195 - 213, 1999 
ajuda com a bibliografia sobre primatas; aos barqueiros e mateiros pelo auxilio de campo e à SOS-Amazônia/The Nature Conservancy/IBAMA pelo convite em participar do projeto e pelo apoio prestado.

\section{REFERÊNCIAS BIBLIOGRÁFICAS}

Auricchio, P. 1995. Primatas do Brasil. São Paulo, Ed. Terra Brasilis, 168p.

AYRES, J.M. \& C. AYRES. 1979. Aspectos da caça no alto rio Aripuanã. Acta Amazônica 9 (2): 287-298.

FonseCA, G.A.B. DA; A.B. Rylands; C.M.R. Costa; R.B. Machado \& Y.L.R. LEITE. 1994. Livro Vermelho dos Mamíferos Brasileiros Ameaçados de Extinção. Editores: Belo Horizonte, Fundação Biodiversitas, 459p.

BODMER, R.E. 1995. Susceptibility of Mammals to Overhunting in Amazonia, 292-295. In: J. BissonetTE \& P. KRAUSMAN (Eds). Integrating People and Wildlife for a Sustainable Future. Bethesda, Maryland, The Wildlife Society, $715 \mathrm{p}$.

BODMER, R.E.; T.G. FANG \& L.M. IBANEZ. 1988a. Primates and ungulates: a comparison of susceptibility to hunting. Primate Conservation 9: 79-84.

. 1988b. Ungulate management and conservation in the Peruvian Amazon. Biological Conservation 45: 303-310.

BURNHAN, K.P.; D.R. ANDERSON \& J.L. LAAKE. 1980. Estimation of density from line transect sampling of biological populations. Wildlife Monographs 72: $1-202$.

Carvalho, C.T. 1957. Alguns mamíferos do Acre Ocidental. Bol. Mus. Paraense Emílio Goeldi, Zool. 6: 1-22.

CONSERVATION INTERNATIONAL. 1991. Workshop 90 - Prioridades Biológicas para Preservação na Amazônia. IBAMA/CI/INPA, Mapa.

DAVIS, W.B. 1973. Geographic variation in the fishing bat, Noctilio leporinus. Jour. Mammalogy 54 (4): 862-874.

EMMONS, L.H. 1984. Geographic variation in densities and diversities of non-flying mammals in Amazonia. Biotropica 16 (3): 210-222.

1987. Comparative feeding ecology of felids in a neotropical rainforest. Behav. Ecol. Sociobiol. 20: 271-283.

EMMONS, L.H. \& F. FEER. 1990. Neotropical Rainforest Mammals - A Field Guide. Chicago, University of Chicago Press, 282p.

Galvão, E.; 1951. Panema: uma crença do caboclo amazônico. Revta Mus. Paulista 5: 221-225.

IHERING, H. 1904. O rio Juruá: mamíferos. Revta Mus. Paulista 6: 406-425.

IMAC. 1991. Atlas Ambiental do Estado do Acre. Rio Branco, Instituto de Meio Ambiente do Acre, 48p.

IUCN. 1996. 1996 IUCN Red List of Threatened Animals. Washington, DC, International Union for Conservation of Nature and Natural Resorces, 448p.

MOK, W.Y.; D.E. WILSON; L.A. LACEY \& R.C.C. LUIZÃO. 1982. Lista atualizada de quirópteros da Amazônia Brasileira. Acta Amazônica 12 (4): 817-823.

MYERS, N. 1988. Threatened biotas: hotspot in tropical forests. Environmentalist $8(3): 1-20$. 
Olalla, A.M. 1938. Um viaje a pesquizas zoológicas: Hacia el Rio Juruá, Estado de Amazonas, Brasil - 1936. Revta Mus. Paulista 22: 233-297.

OrCES, V.G. 1944. Sobre la existencia al norte del Amazonas de los generos Atelocynus y Grammogale. Flora 5: 65-67.

PERES, C.A. 1990. Effects of hunting on western Amazonian primates communities. Biological Conservation 54: 47-59.

-. 1996a. Riverine barriers and gene flow in Amazonian saddle-back tamarins. Folia Primatologica 67: 113-124.

1996b. Population status of white-lipped Tayassu peccari and collared peccaries T. tajacu in hunted and unhunted amazonian forests. Biological Conservation 77: 115-123.

1997a. Primate community structure at twenty western Amazonian flooded and unflooded forests. Jour. Trop. Ecol. 13: 381-485.

$1997 \mathrm{~b}$. Effects of habitat quality and hunting pressure on arboreal folivore densities in neotropical forests: a case study of howler monkeys (Alouatta spp.). Folia Primatologica 68: 199-222.

PERES, C.A. \& J.W. TERBorgh. 1995. Amazonian nature reserves: an analysis of the defensibility status of existing conservation units and design criteria for the future. Conservation Biology 9 (1): 34-45.

PMACI. 1994. Diagnóstico Geoambiental e Sócio-Econômico. Projeto de Proteção do Meio Ambiente e das Comunidades Indígenas Área de Influência da BR-364-Trecho Rio Branco, Cruzeiro do Sul. Rio de Janeiro, IBGE \& IPEA, $144 \mathrm{p}$.

Pook, A.G. \& G. PooK. 1981. A field study of the socioecology of the Goeldi's monkey in northern Bolivia. Folia Primatologica 35 (1): 288-312.

Quigley, H.B. \& P.G. CRAwshaw. 1992. A conservation plan for the jaguar Panthera onca in the Pantanal region of Brazil. Biological Conservation 61: 149-157.

RABINOWITZ, A.R. \& B.G. NotTingham. 1986. Ecology and behaviour of the jaguar (Panthera onca) in Belize, Central America. Jour. Zool. 210: 149-159.

RADAMBRASIL. 1977. Folha SB/SC. 18 Javari/Contamana - Levantamento de

Recursos Naturais, 13 (Geologia, geomorfologia, pedologia, vegetação e uso potencial da terra). Rio de Janeiro, Projeto RADAMBRASIL, DNPM, 413p.

RAMIREZ, M. 1988. The Wolly Monkeys, Genus Lagothrix, p.539-575. In: R.A.

MitTermeier (Ed.). Ecology and Behavior of Neotropical Primates.

Washington, D.C., WWF, Vol. 2, 610p.

REDFORD, K.H. 1992. The empty forest. Bioscience 42 (6): 412-422.

Roosmalen, M.G.M. \& L.L. KLeIN. 1988. The Spider Monkeys, Genus Ateles, p.455-537. In: R.A. MitTermeier; A.B. Rylands; A.F. COIMBRA-FilHo \& G.A.B. DA FONSECA (Eds). Ecology and Behavior of Neotropical Primates. Washington, DC, WWF, Vol. 2, 610p.

Rowe, N. 1996. The Pictorial Guide to the Living Primates. East Hampton, Pogonia Press, 263p.

Rylands, A.B. 1991. The Status of Conservation Areas in the Brazilian Amazon. Washington, DC, WWF, 146p.

TADDEI, V.A.; I.M. REZENDE \& D. CAMORA. 1990. Notas sobre uma coleção de 
morcegos de Cruzeiro do Sul, rio Juruá, estado do Acre (Mammalia, Chiroptera). Bol. Mus. Paraense Emílio Goeldi, Zool. 61 (1): 75-79.

VICKERS, W.T. 1984. The faunal components of lowland South American hunting kills. Interciencia 9 (6): 366-376.

-1991. Hunting Yields and Game Composition Over Ten Years in a Amazon Indian Territory, p.53-81. In: J.G. ROBINSON \& K.H. REDFORD (Eds). Neotropical Wildlife Use and Conservation. Chicago, The University of Chicago Press, 520p.

VIEIRA, C.C. 1949. Nova contribuição ao conhecimento dos mamíferos do rio Juruá.

Bol. Mus. Paraense Emílio Goeldi, Zool. 10: 239-274.

1952. Resultados de uma expedição científica ao Território do Acre: mamíferos. Secretaria da Agricultura, Departamento de Zoologia. Papéis Avulsos Depto Zool.11 (2): 21-32.

Voss, R.T. \& L.H. EMmONS. 1996. Mammalian diversity in neotropical lowland rainforests: a preliminary assessment. Bull. Amer. Mus. Nat. Hist. 230: 1-155.

WeBER, W. \& A. RABINOWITZ. 1996. A global perspective on large carnivore conservation. Conservation Biology 10 (4): 1046-1054.

Wilson, D.E. \& D.M. REEDER. 1993. Mammal Species of the World: A Taxomonic and Geographic Reference. Washington, D.C., Smithsonian Institution, 1207p.

Recebido em 06.VIII.1998; aceito em 26.XI.1999. 